\title{
Estimativa de dimensões de bulbo molhado em irrigação por gotejamento superficial aplicando modelo de superfície de resposta
}

\author{
Estimate of wetted bulb dimensions in surface drip irrigation using response surface model
}

\author{
Celsemy Eleutério Maia ${ }^{I^{*}}$ Sérgio Luiz Aguilar Levien ${ }^{\mathrm{I}}$
}

\section{RESUMO}

As informações de profundidade e largura do bulbo molhado na irrigação localizada são importantes tanto para o dimensionamento, quanto para o manejo da irrigação, auxiliando na determinação da quantidade de água aplicada para a planta. Com o objetivo de avaliar o modelo de superfície de resposta, para estimar as dimensões do bulbo molhado em função da vazão do emissor e do tempo de aplicação de água, foram instalados experimentos em seis solos. Os resultados obtidos mostraram que o modelo proposto pode ser utilizado para estimar as dimensões do bulbo molhado.

Palavras-chave: modelagem de água e solo, infiltração de água no solo, irrigação localizada.

\section{ABSTRACT}

The information of depth and width in wetted zone soil under drip irrigation plays great significance in design and management in the irrigation system for the amount of water required to the plant. For that, six experiments were installed in different soils with the objective of evaluating the dimensions of the wet bulb in the drip irrigation depending on the emitter discharged and of the water application time, using the power model. The obtained results showed that the proposed model can be used to estimate the dimensions of the wetted bulb for the all soils.

Key words: soil and water modeling, soil and water infiltration, localized irrigation.

\section{INTRODUÇÃO}

A irrigação localizada é um sistema de irrigação que tem como principais vantagens a aplicação de água perto das raízes das plantas e a economia de água pela maior eficiência de aplicação. Entre os sistemas de irrigação localizada, o gotejamento superficial é o mais comum e baseia-se na aplicação de água na superfície do solo junto à planta. Esse sistema permite molhar o solo, formando um volume de solo molhado, denominado de bulbo molhado ou bulbo úmido, onde se concentram as raízes das plantas. Molhando-se apenas uma porcentagem do solo, as perdas de água por evaporação e percolação são reduzidas, como também a lixiviação dos nutrientes (YOUNGS et al., 1999). Assim, o manejo da irrigação por gotejamento requer conhecimento do volume de solo molhado para evitar a sobreposição excessiva que pode diminuir a eficiência na aplicação de água e fertilizantes, principalmente em solos de textura arenosa que apresentam maior infiltração (KELLER \& BLIESNER, 1990).

Nas últimas décadas, verificou-se um avanço no desenvolvimento de equipamentos, no dimensionamento e no manejo da irrigação localizada. Isso se deve principalmente ao melhor entendimento do movimento de água no solo quando se utiliza um ponto de emissão de água na superfície. Uma das condições para o melhor dimensionamento da irrigação localizada com ponto de emissão superficial é o conhecimento da distribuição da umidade no solo para diferentes vazões dos emissores e tempo de irrigação, sendo que a vazão e o tempo juntamente com o tipo de solo influenciam o movimento de água (LUBANA \& NARDA, 1998). Resultados empíricos e simulados da

IDepartamento de Ciências Ambientais e Tecnológicas, Universidade Federal Rural do Semiárido (UFERSA), 59625-900, Mossoró, RN, Brasil. E-mail: celsemy@ufersa.edu.br.*Autor para correspondência. 
distribuição de água no solo sob irrigação localizada enfatizam duas características práticas: a) quando o solo seco é irrigado, ocorre a formação do volume de solo molhado, em que a frente de molhamento separa o solo seco do solo molhado; b) a maior fração do volume de solo úmido tem um relativo teor uniforme de água. Esses dois fatores são utilizados na fertirrigação, em que a frente de molhamento define o volume de solo onde a planta emite raiz, e o teor de água e a concentração de nutriente na solução do solo podem ser mantidos próximos do ótimo, contribuindo para a maior eficiência na aplicação de fertilizante (BARYOSEF, 1999). No entanto, COELHO et al. (1999) destacam que os altos investimentos necessários para a implantação desse sistema de irrigação poderão não ser compensados se não forem utilizadas técnicas adequadas de manejo de irrigação que visem à racionalização do uso da água e ao aumento da produtividade.

Dentre os fatores que influenciam a formação do bulbo molhado, pode-se destacar o tipo de solo e a sua estratificação, a vazão do emissor e o tempo de irrigação (PIZARRO, 1996). Outros fatores que influenciam as dimensões do bulbo molhado são: estrutura do solo, sistema de irrigação, altura do emissor em relação ao solo, umidade inicial do solo e presença de concreções e cascalho (BELL et al., 1990; FOLCH \& FÁBREGA, 1999). Nos solos mais argilosos, em razão da menor taxa de infiltração, verifica-se a formação de bulbo com maior dimensão horizontal, explicado pela maior influência da capilaridade sobre a gravidade. Em solos que apresentam camadas estratificadas, verificam-se camadas com diferentes porosidades, que afetam o fluxo e a retenção de água e, consequentemente, o bulbo molhado. Isso implica que, quando a frente de molhamento atinge camadas de diferentes texturas, esta se comporta como uma barreira, principalmente em solos que apresentam gradiente textural no perfil do solo. Para diferentes vazões de emissor e para mesmo tempo de irrigação, menores vazões tenderão a formar bulbos molhados mais profundos e com menor raio superficial; já para mesma vazão de emissores quanto maior o tempo de irrigação, maior o raio superficial.

O objetivo deste trabalho foi avaliar as dimensões do bulbo molhado na irrigação por gotejamento superficial em função da vazão do emissor e do tempo de aplicação de água, usando o modelo de superfície de resposta.

\section{MATERIAL E MÉTODOS}

Para uma avaliação do comportamento do bulbo molhado, foi feito um levantamento dos principais solos utilizados na fruticultura irrigada do Rio Grande do Norte, mais precisamente no Agropólo AssuMossoró. A região é caracterizada, segundo a classificação de Gaussen, com bioclima da região de 4ath tropical quente de seca a acentuada, com índice xerotérmico entre 200 e 150 e sete a oito meses secos. Segundo a classificação de Köppen, o clima da região é do tipo BSwh, isto é, seco, muito quente e com estação chuvosa no verão, atrasando-se para o outono e apresentando temperatura média anual de $27,4^{\circ} \mathrm{C}$, precipitação pluviométrica anual bastante irregular, com média de 673,9mm e umidade relativa de 68,9\%(CARMO FILHO et al., 1991).

Foram selecionados seis solos com diferentes características físicas e mineralógicas. De acordo com EMBRAPA (1999), os solos selecionados para o referido estudo foram classificados como Luvissolo Crômico, Argissolo Vermelho-Amarelo, Latossolo Vermelho, Cambissolo Háplico, Neossolo Quartizarênico e Neossolo Flúvico. Antes da montagem do sistema de irrigação, os solos foram preparados de forma a simular as reais condições de plantio, com aração e passagem da grade de disco de forma cruzada.

Para determinar com maior exatidão o tamanho do bulbo molhado (diâmetro da área molhada e profundidade), foi desenvolvido um sistema de irrigação portátil, em que foram dispostos oito emissores por linha, distribuídos aleatoriamente na parcela, ao longo de uma tubulação de polietileno de 16 mm de diâmetro, abastecida por dois depósitos de água (1000L e 500L). Para regular a vazão e a pressão, foram utilizados reservatórios reguladores, conectados aos primeiros, que mantinham um nível constante por meio de um sistema de bóia. As diferentes vazões foram obtidas pelos diferentes comprimentos dos microtubos inseridos na tubulação de polietileno. Estes, juntamente com a carga hidráulica constante, geraram diferentes vazões (1; 2; 4 e 8L h $\left.{ }^{-1}\right)$ em suas saídas e eram fechados quando cada tempo de irrigação era concluído. O espaçamento entre os emissores foi estabelecido para 
que não ocorresse sobreposição na formação dos bulbos, ou seja, interferência dos emissores laterais.

Os experimentos foram instalados em delineamento em blocos inteiramente casualizados, no esquema de parcela subdividida, com três repetições, cujos tratamentos foram compostos pelos fatores tempo de aplicação de água e vazão do emissor, sendo a parcela formada pelo tempo e a subparcela formada pela vazão. As repetições constaram de duas linhas de irrigação, com quatro diferentes tempos (1; 2; 4 e 7h) e, dentro de cada tempo, as vazões de $1 ; 2 ; 4$ e $8 \mathrm{~L} \mathrm{~h}^{-1}$ obtidas por microtubos de tamanhos diferenciados que proporcionam as vazões desejadas sob as mesmas condições de carga hidráulica.

Para cada vazão, após os tempos prédeterminados de 1; 2; 4 e 7h, foram abertas trincheiras no centro do bulbo molhado, abaixo do emissor, onde foram realizadas as medições das dimensões do bulbo com fita métrica, com precisão de 1,0mm. Para avaliar o efeito da vazão do emissor e do tempo de aplicação de água na formação do bulbo molhado, avaliaram-se as seguintes características: diâmetro superficial $\left(D_{s}\right)$, diâmetro máximo $\left(D_{\max }\right)$, profundidade máxima $\left(Z_{\max }\right)$ e profundidade onde ocorre $D_{\max }\left(Z_{D \max }\right)$. Além dessas medidas, foram medidos os diâmetros a cada 5 ou $10 \mathrm{~cm}$ de profundidade para avaliação do formato do bulbo molhado. Para avaliar as dimensões do bulbo molhado em função de $q$ e $t$, foram realizadas análises de regressão a fim de avaliar o comportamento do bulbo pelo modelo de superfície de resposta. Para tanto, observou-se, em outros trabalhos, que as dimensões do bulbo em função de $t$ ou $q$ isoladamente podem ser estimadas com o modelo potencial (THORBURN et al., 2003; LI et al., 2004); assim, a partir de $y=\alpha_{1} . t \beta^{1}$ e $y=$ $\alpha_{1} . q \beta^{1}$ e da mesma medida do bulbo, tem-se $y=$ $\alpha_{1} \cdot t \beta^{1} \cdot \alpha_{2} \cdot q \beta^{2}$. Sendo $\alpha=\alpha_{1} \cdot \alpha_{2}$, tem-se $y=\alpha \cdot t \beta^{1} \cdot q \beta^{2}$ ou $y=a \cdot t^{b} \cdot q^{c}$, em que t é o tempo de aplicação de água (h), q é a vazão do emissor ( $\left(\mathrm{h}^{-1}\right)$ e a, b e c são parâmetros do modelo.

Para avaliar o uso de modelo de superfície de resposta para estimativa das dimensões do bulbo em função de $q$ e $t$, foi utilizada a metodologia proposta por GAUCH et al. (2003), baseada no Quadrado Médio dos Desvios (MSD), que é decomposto em Quadrado da Bias (SB), Quadrado Médio para Declividade não Unitária (NU) e Quadrado Médio para Falta de
Correlação (LC), sendo MSD = SB+NU+LC. O SB está relacionado com o intercepto da reta ajustada entre os valores estimados e observados, o NU está relacionado com a declividade da reta e o LC, com a correlação entre os dados observados e estimados pelos modelos e que quanto menor o valor do MSD, melhor será o modelo.

\section{RESULTADOS E DISCUSSÃO}

Pelo modelo de superfície de resposta ajustado aos dados (Tabela 1), observa-se que, com exceção de $Z_{\max }, Z_{\text {Dmax }}$ e $D_{\text {max }}$, para o Neossolo Flúvico, os coeficientes de determinação foram superiores a $90 \%$ para todas as dimensões dos solos avaliados. Levandose em consideração que as dimensões do bulbo podem ser estimadas isoladamente em função de $q$ ou $t$ e utilizando-se o modelo potencial, uma das vantagens da superfície de resposta é que apenas 24 equações são suficientes para abranger todas as combinações de $t$ e $q$ do experimento, ao contrário do modelo potencial, em que esse número seria de 192 equações.

Os valores estimados das dimensões do bulbo molhado em função dos valores observados indicam que o modelo de superfície de resposta estimou bem essas dimensões e, além dos altos coeficientes de determinação, observa-se que os coeficientes angulares e os interceptos da reta ajustada foram estatisticamente iguais a 1 e 0 , respectivamente (Figura 1 ).

Ao se fazer uma simulação para a cultura do melão com os resultados obtidos, para uma profundidade do bulbo molhado de $40 \mathrm{~cm}$ e utilizandose a equação $\mathrm{Z}_{\text {max }}=f(t, q)$, tem-se o modelo $\mathrm{t}=f(q)$, que combina os valores de $t$ e $q$ para obter a profundidade máxima de $40 \mathrm{~cm}$. Utilizando-se o critério de $\frac{d t}{d q}=-1$ para melhor combinação de $t$ e $q$, obtémse o valor de $q$, com o qual, sendo substituído em $\mathrm{t}=$ $f(q)$, determina-se o tempo $t$ para atingir $Z_{\text {max }}$ igual a $40 \mathrm{~cm}$. Os valores de $D_{\max }, D_{s}$ e $Z_{D \max }$ foram obtidos pela substituição dos valores de $t$ e $q$ em $\mathrm{D}_{\max }=f(t, q), \mathrm{D}_{\mathrm{s}}=$ $f(t, q)$ e $\mathrm{Z}_{\text {max }}=f(t, q)$, respectivamente. A partir do critério proposto por KELLER \& BLIESNER (1990), multiplicando-se o valor de $D_{\max }$ por 0,8 , tem-se o espaçamento entre os emissores.

Assim, sem considerar o Neossolo Flúvico, os valores de $q$ para um $Z_{\text {max }}$ de $40 \mathrm{~cm}$ variaram de 3,019

Ciência Rural, v.40, n.6, jun, 2010. 
Tabela 1 - Parâmetros do modelo de superfície de resposta $\left(y=a \cdot t^{b} \cdot q^{c}\right)$ para profundidade máxima $\left(Z_{\text {max }}\right)$, diâmetro superficial $\left(\mathrm{D}_{\mathrm{s}}\right)$, profundidade onde ocorre o diâmetro máximo $\left(Z_{D \max }\right)$ e diâmetro máximo $\left(D_{\max }\right)$, para os solos estudados.

\begin{tabular}{|c|c|c|c|c|c|}
\hline Solo & Dimensões & a & $b$ & c & $\mathrm{R}^{2}$ \\
\hline \multirow{4}{*}{ Luvissolo Crômico } & $Z_{\max }$ & 14,5658 & 0,4196 & 0,3553 & 0,9710 \\
\hline & $D_{s}$ & 25,1965 & 0,2100 & 0,2659 & 0,9805 \\
\hline & $Z_{D \max }$ & 3,8550 & 0,5547 & 0,4382 & 0,9524 \\
\hline & $D_{\max }$ & 24,8723 & 0,3025 & 0,3361 & 0,9929 \\
\hline \multirow{4}{*}{ Argissolo Vermelho-Amarelo } & $Z_{\max }$ & 16,3093 & 0,4047 & 0,4260 & 0,9864 \\
\hline & $D_{s}$ & 24,8700 & 0,2094 & 0,2640 & 0,9833 \\
\hline & $Z_{D \max }$ & 4,8463 & 0,5727 & 0,4120 & 0,9766 \\
\hline & $D_{\max }$ & 24,7273 & 0,3556 & 0,3107 & 0,9678 \\
\hline \multirow{4}{*}{ Cambissolo Háplico } & $Z_{\max }$ & 14,6370 & 0,4069 & 0,4001 & 0,9515 \\
\hline & $D_{s}$ & 26,4790 & 0,2441 & 0,2742 & 0,9165 \\
\hline & $Z_{D \max }$ & 2,0712 & 0,7882 & 0,5162 & 0,9517 \\
\hline & $D_{\max }$ & 24,6262 & 0,3463 & 0,3273 & 0,9851 \\
\hline \multirow{4}{*}{ Neossolo Quartzarênico } & $Z_{\max }$ & 15,6967 & 0,4098 & 0,3763 & 0,9280 \\
\hline & $D_{s}$ & 25,9539 & 0,1927 & 0,2251 & 0,9634 \\
\hline & $Z_{\text {Dmax }}$ & 3,6340 & 0,6502 & 0,5208 & 0,9518 \\
\hline & $D_{\max }$ & 24,6603 & 0,3435 & 0,3238 & 0,9804 \\
\hline \multirow{4}{*}{ Latossolo Vermelho } & $Z_{\max }$ & 13,9914 & 0,4307 & 0,4635 & 0,9696 \\
\hline & $D_{s}$ & 22,5462 & 0,1945 & 0,2225 & 0,9255 \\
\hline & $Z_{\text {Dmax }}$ & 5,4593 & 0,5088 & 0,4430 & 0,9855 \\
\hline & $D_{\max }$ & 23,4125 & 0,3329 & 0,3265 & 0,9637 \\
\hline \multirow{4}{*}{ Neossolo Flúvico } & $Z_{\max }$ & 11,7550 & 0,2548 & 0,2096 & 0,6372 \\
\hline & $D_{s}$ & 21,0619 & 0,3357 & 0,4086 & 0,9564 \\
\hline & $Z_{\text {Dmax }}$ & 0,0047 & $-3,2903$ & 4,3771 & 0,8350 \\
\hline & $D_{\max }$ & 17,8365 & 0,4978 & 0,3609 & 0,8075 \\
\hline
\end{tabular}

a 3,446 $\mathrm{L} \mathrm{h}^{-1}$, para o Argissolo Vermelho-Amarelo e o Cambissolo Háplico, respectivamente, e o tempo de irrigação variou de 2,864 a 3,975h, para o Argissolo Vermelho-Amarelo e o Luvissolo Crômico, respectivamente (Tabela 2). Os valores obtidos para o Neossolo Flúvico não foram comparados em razão da profundidade máxima observada no campo na maior vazão e do tempo de aplicação de água ter sido de aproximadamente $30 \mathrm{~cm}$. Desse modo, os dados desse solo apresentados na tabela 2 são extrapolações, ou seja, os resultados estão fora do espaço experimental, e a principal explicação para os valores elevados de $t$ e $q$ para se alcançar os $40 \mathrm{~cm}$ é a baixa condutividade hidráulica desse solo.

Na avaliação do volume de água aplicado para a formação do bulbo, observa-se que, para atingir os $40 \mathrm{~cm}$ de profundidade, o volume de água foi diferente para os solos estudados, sendo maior no Luvissolo Crômico e menor no Argissolo Vermelho-Amarelo, sem considerar o Neossolo Flúvico. Apesar do volume de água aplicado para atingir um $Z_{\max }$ de $40 \mathrm{~cm}$ ter variado entre os solos, quando se verifica a lâmina de água aplicada pelo sistema $\left(\mathrm{mm} \mathrm{h}^{-1}\right)$, os valores se aproximam, com média de $3,788 \mathrm{~mm} \mathrm{~h}^{-1}$. Isso se deve a diferença no espaçamento entre os emissores, considerado como sendo igual a $0,8 D_{\max }$, e este difere entre os solos.

Na avaliação do modelo da superfície de resposta pelo MSD, apenas para o Neossolo Flúvico os valores foram elevados, principalmente para $D_{\max }$, $D_{s}$ e $Z_{\text {Dmax }}$. Para os demais solos, observa-se que houve bom ajuste do modelo aos dados observados e que o principal componente afetado é a falta de correlação, porém com valores todos superiores a 93\%, com exceção do Neossolo Flúvico (Tabela 3). 

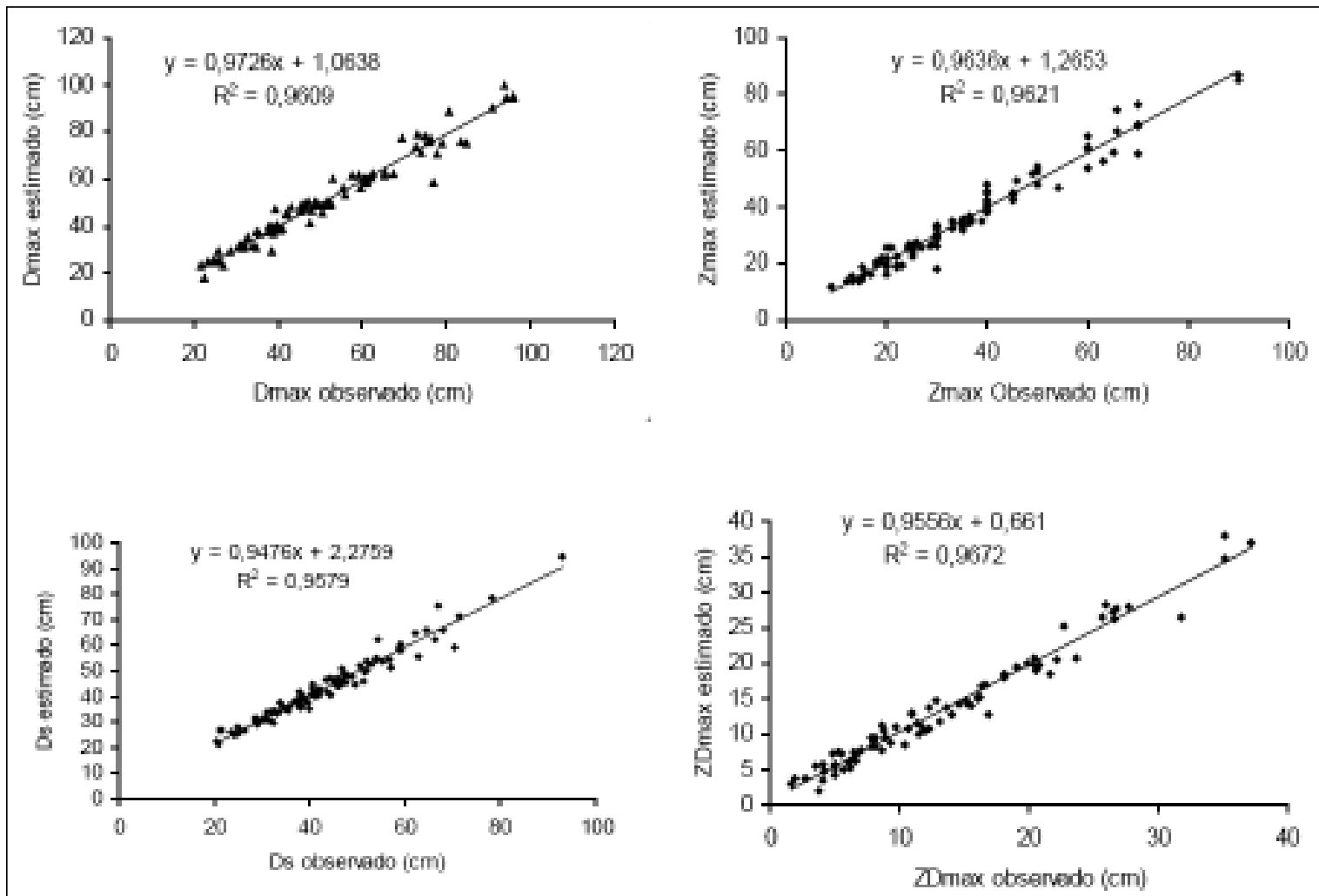

Figura 1 - Valores estimados em função de valores observados para diâmetro máximo $\left(D_{\text {max }}\right)$, profundidade máxima $\left(Z_{\text {max }}\right)$, diâmetro superficial $\left(D_{s}\right)$ e profundidade onde ocorre $D_{\max }\left(Z_{D \max }\right)$, usando o modelo superfície de resposta, para os solos estudados.

\section{CONCLUSÃO}

O modelo superfície de resposta pode ser utilizado para estimar os valores de $D_{\max }, Z_{\max }, D_{s}$ e
$Z_{D \max }$ em função da vazão do emissor e tempo de aplicação de água na irrigação por gotejamento superficial.

Tabela 2 - Valores de vazão do emissor ( $q$, em L h $\left.{ }^{-1}\right)$, tempo de aplicação de água ( $t$, em h) e volume de água aplicado $\left(V_{a p}\right.$, em L) usados para obter a profundidade do bulbo molhado de $40 \mathrm{~cm}$ e valores obtidos de diâmetro máximo $\left(\mathrm{D}_{\max }\right)$, diâmetro superficial $\left(\mathrm{D}_{\mathrm{s}}\right)$ e profundidade onde ocorre $D_{\max }\left(\mathrm{Z}_{\mathrm{Dmax}}\right)$, para os solos estudados.

\begin{tabular}{lcccrrr}
\hline & Luvissolo Crômico & $\begin{array}{c}\text { Argissolo } \\
\text { Vermelho- } \\
\text { Amarelo }\end{array}$ & $\begin{array}{c}\text { Cambissolo } \\
\text { Háplico }\end{array}$ & $\begin{array}{c}\text { Neossolo } \\
\text { Quartzarênico }\end{array}$ & $\begin{array}{c}\text { Latossolo } \\
\text { Vermelho }\end{array}$ & Neossolo Flúvico \\
\hline$q(\mathrm{Z}=40 \mathrm{~cm})$ & 3,366 & 3,019 & 3,446 & 3,144 & 3,354 & 12,549 \\
$t(\mathrm{Z}=40 \mathrm{~cm})$ & 3,975 & 2,868 & 3,505 & 3,424 & 3,117 & 15,255 \\
$V_{a p}(\mathrm{~L})$ & 13,378 & 8,660 & 12,076 & 10,765 & 10,452 & 191,424 \\
$D_{\max }{ }^{*}(\mathrm{~cm})$ & 56,777 & 50,696 & 57,000 & 54,534 & 50,746 & 172,557 \\
$D_{s}^{(*)}(\mathrm{cm})$ & 46,492 & 41,514 & 50,490 & 42,577 & 36,817 & 147,763 \\
$Z_{\operatorname{Dax}}{ }^{*}(\mathrm{~cm})$ & 14,106 & 13,970 & 10,541 & 14,690 & 16,638 & 0,039 \\
$0,8 D_{\max }(\mathrm{cm})$ & 45,422 & 40,557 & 45,600 & 43,627 & 40,597 & 138,045 \\
$\operatorname{TAS}^{(* *)}$ & 3,705 & 3,722 & 3,778 & 3,603 & 4,131 & 4,545 \\
LAA $^{(* * *)}$ & 14,727 & 10,676 & 13,242 & 12,337 & 12,873 & 69,334 \\
\hline
\end{tabular}

${ }^{(*)}$ Estimado pelo modelo de superfície de resposta.

${ }^{(* *)}$ Taxa de aplicação de água pelo sistema $\left(\mathrm{mm} \mathrm{h}^{-1}\right)$ considerando o espaçamento entre linhas de $2 \mathrm{~m}$ e a distância entre emissores de $0,8 D_{\max }$.

${ }^{(* * *)}$ Lâmina de água aplicada pelo sistema de irrigação (mm). 
Tabela 3 - Valores de MSD, RMSD e seus componentes SB, NU e LC e coeficiente de determinação obtidos na avaliação dos valores estimados e observados pelo modelo superfície de resposta, para profundidade máxima $\left(Z_{\max }\right)$, diâmetro máximo $\left(D_{\max }\right)$, diâmetro superficial $\left(D_{s}\right)$ e profundidade onde ocorre $D_{\max }\left(Z_{D \max }\right)$, em função do tempo de aplicação de água $(t)$ e da vazão do emissor $(q)$, para os solos estudados.

\begin{tabular}{|c|c|c|c|c|c|c|}
\hline Solo & MSD & RMSD & SB & NU & LC & $\mathrm{R}^{2}$ \\
\hline \multicolumn{7}{|c|}{ - } \\
\hline LC & 6,0713 & 2,4640 & 0,0024 & 0,0144 & 6,0545 & 0,9711 \\
\hline AVA & 4,7124 & 2,1708 & 0,0069 & 0,0350 & 4,6705 & 0,9865 \\
\hline $\mathrm{CH}$ & 13,1058 & 3,6202 & 0,0164 & 0,0885 & 13,0009 & 0,9518 \\
\hline NQ & 20,4910 & 4,5267 & 0,0140 & 0,0805 & 20,3965 & 0,9283 \\
\hline LVA & 10,4310 & 3,2297 & 0,0501 & 0,2174 & 10,1635 & 0,9704 \\
\hline NF & 6,9807 & 2,6421 & 0,3505 & 0,0416 & 6,5886 & 0,7995 \\
\hline Todos & 10,3337 & 3,2146 & 0,0056 & 0,0034 & 10,3247 & 0,9662 \\
\hline & & & $---D_{\max }-$ & & & \\
\hline LC & 2,1173 & 1,4551 & 0,0018 & 0,0148 & 2,1007 & 0,9929 \\
\hline AVA & 11,2125 & 3,3485 & 0,0002 & 0,0018 & 11,2105 & 0,9677 \\
\hline $\mathrm{CH}$ & 5,3537 & 2,3138 & 0,0014 & 0,0102 & 5,3421 & 0,9851 \\
\hline NQ & 6,6616 & 2,5810 & 0,0042 & 0,0326 & 6,6248 & 0,9805 \\
\hline LVA & 11,5335 & 3,3961 & 0,0161 & 0,1323 & 11,3851 & 0,9642 \\
\hline NF & 90,8018 & 9,5290 & 10,0067 & 3,6131 & 77,1820 & 0,8440 \\
\hline Todos & 20,5481 & 4,5330 & 0,2424 & 0,1815 & 20,1242 & 0,9440 \\
\hline LC & 2,8669 & 1,6932 & 1,1854 & 0,3948 & 1,2867 & 0,9904 \\
\hline AVA & 2,8772 & 1,6962 & 0,0644 & 0,0782 & 2,7346 & 0,9752 \\
\hline $\mathrm{CH}$ & 14,4673 & 3,8036 & 0,0454 & 1,7363 & 12,6856 & 0,9415 \\
\hline NQ & 4,1318 & 2,0327 & 0,6410 & 0,2150 & 3,2758 & 0,9657 \\
\hline LVA & 6,8041 & 2,6085 & 1,6113 & 0,1273 & 5,0655 & 0,9310 \\
\hline $\mathrm{NF}$ & 90,6454 & 9,5208 & 0,7581 & 0,6771 & 89,2102 & 0,8034 \\
\hline Todos & 19,55851 & 4,4225 & 0,5597 & 0,0094 & 18,9894 & 0,9016 \\
\hline & & & $---Z_{D \max }$ & & & \\
\hline LC & 2,1833 & 1,4776 & 0,0100 & 0,0355 & 2,1378 & 0,9534 \\
\hline AVA & 1,5309 & 1,2373 & 0,0009 & 0,0032 & 1,5268 & 0,9766 \\
\hline $\mathrm{CH}$ & 2,3670 & 1,5385 & 0,0009 & 0,0018 & 2,3643 & 0,9518 \\
\hline NQ & 4,3339 & 2,0818 & 0,0184 & 0,0474 & 4,2681 & 0,9525 \\
\hline LVA & 1,0365 & 1,0181 & 0,0032 & 0,0128 & 1,0205 & 0,9857 \\
\hline $\mathrm{NF}$ & - & - & - & - & - & - \\
\hline Todos & 2,2904 & 1,5134 & 0,0034 & 0,0097 & 2,2773 & 0,9672 \\
\hline
\end{tabular}

LC - Luvissolo Crômico, AVA - Argissolo Vermelho-Amarelo, CH - Cambissolo Háplico, NQ - Neossolo Quartzarênico, LVA - Latossolo Vermelho, NF - Neossolo Flúvico

Quadrado Médio dos Desvios (MSD), raiz do Quadrado Médio dos Desvios (RMSD), Quadrado da Bias (SB), Quadrado Médio para Declividade não Unitária (NU) e Quadrado Médio para Falta de Correlação (LC).

\section{REFERÊNCIAS}

BAR-YOSEF, B. Advances in fertigation. Advances in Agronomy, v.65, p.1-77, 1999.

BELL, J.P. et al. Soil water status: a concept for characterizing soil water conditions beneath a drip irrigated row crop. Agricultural Water Management, v.17, p.171-187, 1990. Disponível em: <http:/ /www.sciencedirect.com/science?_ob=ArticleURL\&_udi=B6T3X4888 H6H-1V\&_user $=684796 \&$ _coverDate $=01 \% 2$ F 3 $1 \% 2 \mathrm{~F} 1990$ \&_rdoc=16\&_fmt=high\&_orig=browse\&_srch=docinfo(\%23toc\%234958\%231990\%23999829998\%23411130\%23FL P\%23display\%23Volume)\&_cdi=4958\&_sort=d\&_docanch or $=\& \_c t=35 \& \_$acct $=C 000036759 \&$ \& version $=1 \&$ _urlVe rsion $=0 \& \_$_userid $=684796 \& m d 5=b 89 a 4 e e d 25 d 8637586 c 15 f c 600 c 25 \mathrm{db} 4>$. Acesso em: 01 abr. 2010. doi: 10.1016/0378-3774(90)90064-6.

CARMO FILHO, F. et al. Dados meteorológicos de Mossoró (Jan. de 1988 à Dez. de 1990). Mossoró: ESAM/FGD, 1991. 121p. (Coleção Mossoroense).

COELHO, E.F. et al. Avaliação de parâmetros hidráulicos para modelos de distribuição de água no solo sob gotejamento. Pesquisa Agropecuária Brasileira, v.34, n.4, p.651-657, 1999.

Ciência Rural, v.40, n.6, jun, 2010. 
EMBRAPA. Centro Nacional de Pesquisas de Solos. Sistema brasileiro de classificação de solos. Rio de Janeiro, 1999. 412p.

FOLCH, J.R.G.; FÁBREGA, J.A.G. El volumen húmedo del suelo en el riego localizado: importancia y evaluación. In: CARPENA, R.M. et al. (Eds). Estudios de la zona no saturada del suelo. Tenerife: ICIA, 1999. p.11-17.

KELLER, J.; BLIESNER, R.D. Sprinkle and trickle irrigation. New York: AVI Book, 1990. 640p.

LI, J. et al. Wetting patterns and nitrogen distributions as affected by fertigation strategies from a surface point source. Agricultural Water Management, v.67, p.89-104, 2004. Disponivel em: <http:/ /www.sciencedirect.com/science?_ob=ArticleURL\&_udi=B6T3X4 C53PYC-2\&_user $=684796 \&$ \& coverDate $=06 \% 2 \mathrm{~F} 15 \% 2 \mathrm{~F}$ $2004 \&$ \&doc $=3 \&$ \&mt $=$ high \&_orig =browse \&_srch=doc info(\%23toc\%234958\%232004\%23999329997\%2350 1774\%23FLA\%23display\%23Volume)\&_cdi=4958\&_sort=d\& _docanchor $=$ \&_ct $=7 \&$ \&_act $=\mathrm{C} 000036759$ \&_version $=1 \&$ \&urlV ersion=0\&_userid=684796\&md5=16cdd650450ea83124060ca769e95d44>. Acesso em: 01 abr. 2010. doi: 10.1016/j.agwat.2004.02.002.

LUBANA, P.P.S.; NARDA, N.K. Soil water dynamics model for trickle irrigated tomatoes. Agricultural Water Management, v.37, p.145-161, 1998. Disponivel em: <http:// www.sciencedirect.com/science?_ob=ArticleURL\&_udi=B6T3X3VGM9SB-5\&_user $=684796 \&$ \&_coverDate $=07 \% 2$ F01\%2F19 98\&_rdoc $=5 \&$ \&_fmt $=$ high\&_orig =browse \&_srch $=$ doc info(\%23toc\%234958\%231998\%23999629997\%2346069\%2
3FLA\%23display\%23Volume)\&_cdi=4958\&_sort=d\&_docanchor=\&_ct $=8 \& \_$_acct $=$C000036759\&_version $=1 \&$ _urlVersion $=0$ \&_userid $=68$ 4796\&md5=e35f0b7679e8e90c59ff08212a4dc19d >. Acesso em: 01 abr. 2010. doi: 10.1016/S0378-3774(98)00035-3.

PIZARRO, F. Riegos localizados de alta frecuencia (RLAF): goteo, microaspersión, exudación. Madrid: MundiPrensa, 1996. 511p.

SCHWARTZMAN, M.; ZUR, B. Emitter spacing and geometry of wetted soil volume. Journal of Irrigation and Drainage Engineering, v.112, n.3, p.242-253, 1986.

THORBURN, P.J. et al. Soil-dependent wetting from trickle emitters: implications for system design and management. Irrigation Science, v.22, p.121-127, 2003. Disponivel em <http://www.springerlink.com.w10013.dotlib.com.br/content/ 14g42wquu4w0896r/fulltext.html>. Acesso em: 01 abr. 2010. doi: 10.1007/s00271-003-0077-3.

van GENUCHTEN, M.T. A closed-form equation for predicting the hydraulic conductivity of unsaturated soils. Soil Science Society of American Journal, v.44, p.892-898, 1980.

YOUNGS, E.G. et al. Surface ponding of coarse-textured soil under irrigation with a line of surface emitters. Journal of Agricultural Engineering Research, v.73, p.95-100, 1999.

ZUR, B. Wetted soil volume as a design objective in trickle irrigation. Irrigation Science, v.16, p.101-105, 1996. 